This is a postprint version of the following published document:

Aptekarev A. I.; López Lagomasino, G.; Martínez-Finkelshtein, A. (2015). "Strong asymptotics for the Pollaczek multiple orthogonal polynomials". Doklady Mathematics, November, v. 92, Issue 3, pp. 709-713.

DOI: $10.1134 / \mathrm{S} 1064562415060216$

Proyectos:

MTM2012-36732-C03-01

MTM2011-28952-C02-01

(C) Springer 2015 


\title{
Strong Asymptotics for the Pollaczek Multiple Orthogonal Polynomials ${ }^{1}$
}

\author{
A. I. Aptekarev ${ }^{a}$, G. López Lagomasino ${ }^{b}$, and A. Martínez-Finkelshtein ${ }^{c}$
}

\begin{abstract}
The asymptotic properties of multiple orthogonal polynomials with respect to two Pollaczek weights with different parameters are considered. This set of weights is a Nikishin system generated by two measures with unbounded supports; moreover, the second measure is discrete. During the last years, multiple orthogonal polynomials with respect to Nikishin systems of this type have found wide applications in the theory of random matrices. Strong asymptotic formulas for the polynomials under consideration are obtained by means of the matrix Riemann-Hilbert method.
\end{abstract}

DOI: $10.1134 / \mathrm{S} 1064562415060216$

In [1] Pollaczek introduced a family of polynomials defined by the recurrent relations (for parameters $\lambda>0$, $\varphi \in(0, \pi))$ :

$$
\begin{gathered}
n P_{n}(x)=2((n-1+\lambda) \cos \varphi+x \sin \varphi) P_{n-1}(x) \\
-(n-2+2 \lambda) P_{n-2}(x), \quad n \in \mathbb{N},
\end{gathered}
$$

with initial conditions $P_{-1}(x)=0$ and $P_{0}=1$. These polynomials are orthogonal

$$
\int_{\mathbb{R}} x^{v} P_{n}(x) w^{(\lambda, \varphi)}(x) d x=0, \quad v=0,1, \ldots, n-1
$$

with respect to the weight functions

$$
w^{(\lambda, \varphi)}(x):=|\Gamma(\lambda+i x)|^{2} e^{(2 \varphi-\pi) x}, \quad x \in \mathbb{R} .
$$

In the last decade, interest has been aroused concerning the family of multiple orthogonal polynomials $\tilde{P}_{n}(x)=x^{p n}+\ldots$ :

$$
\begin{gathered}
\int_{\mathbb{R}} x^{v} \tilde{P}_{n}(x) w_{j}(x) d x=0, \quad v=0,1, \ldots, n-1, \\
j=1,2, \ldots, p,
\end{gathered}
$$

with respect to Pollaczek weights (1). For example, the formal properties of family (2) for the system of weights

\footnotetext{
${ }^{1}$ The article was translated by the authors.

${ }^{a}$ Keldysh Institute of Applied Mathematics, Russian Academy of Sciences, Moscow, Russia

${ }^{b}$ Department of Mathematics, Universidad Carlos III de Madrid, Leganés, Spain

${ }^{c}$ Department of Mathematics, University of Almeria, Almeria, Spain

e-mail:aptekaa@keldysh.ru,lago@math.uc3m.es, andrei@ual.es
}

$$
w_{j}(x):=w^{\left(\lambda, \varphi_{j}\right)}(x), \quad x \in \mathbb{R}, \quad j=1,2, \ldots, p
$$

were studied in $[2,13]$. For two weights

$$
\begin{aligned}
& w_{1}(x):=w^{(1, \pi / 2)}(x)=\frac{x}{3 \sinh \pi x}, \\
& w_{2}(x):=w^{(1 / 2, \pi / 2)}(x)=\frac{x}{3 \cosh \pi x}
\end{aligned}
$$

Sorokin $[2,13]$ investigated the weak asymptotics of scaled polynomials (2), (4)

$$
\tilde{Q}_{n}(z):=\frac{\tilde{P}_{n}(n z)}{n^{2 n}}, \quad w_{j, n}(x):=w_{j}(n x), \quad j=1,2
$$

(in what follows, we set $p=2$ in (2)). More specifically, he found the main term of the asymptotics

$$
\sqrt[n]{\left|\tilde{Q}_{n}(z)\right|} \rightrightarrows|\Phi(z)|, \quad n \rightarrow \infty, \quad z \in K \Subset \overline{\mathbb{C}} \backslash \mathbb{R}, \text { (6) }
$$

and the limiting zeros distribution

$$
v_{\tilde{Q}_{n}} \rightarrow{ }^{*} \lambda, \quad n \rightarrow \infty, \quad \text { where } \quad v_{\tilde{Q}_{n}}:=\frac{1}{n} \sum_{v_{\tilde{Q}_{n}}(x)=0} \delta_{x} .
$$

In this paper we refine Sorokin's results (see [3]), namely, formulas for strong asymptotics and outline their proof.

\section{RANDOM MATRICES \\ AND NIKISHIN SYSTEMS}

In the last decade, interest in the multiple orthogonal polynomials (2) has been related to their natural appearance in some ensembles of random matrices (see, for example, $[4-9,12,13]$ ). Note that the polynomial $\tilde{P}_{n}$ is the mathematical expectation (average) of the characteristic polynomial of a random matrix; therefore (under a proper scaling) the limiting zero counting measure $\lambda$ describes the average of the eigenvalue distribution density. 
A common feature of the systems of weights $\left\{w_{j}\right\}_{j=1}^{2}$ from (2) used in applications to random matrix ensembles was the fact that $w_{1}$ and $w_{2}$ were special entire functions and their ratio $\frac{w_{2}}{w_{1}}$ was a meromorphic function with poles located outside the common support of the orthogonality measures $w_{j}(x) d x$, $j=1,2$.

Two measures $\{d \mu, \hat{\sigma} d \mu\}$ with a common connected support $\Delta:=\operatorname{supp} \mu$ are called a Nikishin system if the derivative of the second measure with respect to the first one is the Cauchy transform of a third measure $\sigma$ with a support supp $\sigma \subseteq \delta$, where $\sigma$ is the union of a finite number of connected sets nonintersecting with $\Delta$, i.e.,

$$
\begin{gathered}
\{d \mu, \hat{\sigma} d \mu\} \in \mathcal{N} \Leftrightarrow \hat{\sigma}(z)=\int_{\delta} \frac{d \sigma(x)}{z-x}, \\
\delta \cap \Delta=\phi .
\end{gathered}
$$

This notion was introduced by Nikishin in 1980 [10] for (an arbitrary number) $p$ of measures with a common support $\Delta \Subset \mathbb{R}$. Nowadays, Nikishin systems and their generalizations are the basic class of weights for multiple orthogonal polynomials (see, for example, $[11,12,14])$.

The multiple orthogonal polynomials $\tilde{P}_{n}(x)=$ $x^{2 n}+\ldots$ with respect to the Nikishin system $\{d \mu, \hat{\sigma} d \mu\}$ are also characterized by usual orthogonality relations (see, for example, $[11,14]$ ). There exists a polynomial $\tilde{P}_{n, 2}(x)=x^{n}+\ldots$ with zeros on $\delta$ such that

$$
\begin{gathered}
\int_{\Delta} x^{v} \tilde{P}_{n}(x) \frac{d \mu(x)}{\tilde{P}_{n, 2}(x)}=0, \quad v=0,1, \ldots, 2 n-1, \\
\int_{\delta} t^{v} \tilde{P}_{n, 2}(t) \frac{1}{\tilde{P}_{n}(t)} \int_{\Delta} \frac{\tilde{P}_{n}^{2}(x)}{\tilde{P}_{n, 2}(x)} \frac{d \mu(x)}{x-t} d \sigma(t)=0, \\
v=0,1, \ldots, n-1 .
\end{gathered}
$$

For the Pollaczek multiple orthogonal polynomials (4), (2), we have

$$
\begin{gathered}
\Delta:=\mathbb{R}, \quad \delta:=i(\mathbb{R} \backslash\{0\}), \\
d \mu(x)=\frac{x d x}{\sinh (\pi x)}, \quad \sigma=\frac{4}{\pi} \sum_{k \in \mathbb{Z}} \delta_{i(2 k+1)} .
\end{gathered}
$$

The system of orthogonality relations (8), (9) leads (see $[13,14])$ to equilibrium conditions characterizing the limiting zeros counting measure (7) of scaled polynomials (5). There exists a unique pair of measures $|\lambda|=2,\left|\lambda_{2}\right|=1,\left|\lambda_{2}\right| \leq l$, such that

$$
\begin{gathered}
2 \mathscr{P}^{\lambda}(x)-\mathscr{P}^{\lambda_{2}}(x)+\pi|x| \begin{cases}=\gamma_{1}, & x \in \operatorname{supp} \lambda \subset \mathbb{R}, \\
\geq \gamma_{1}, & x \in \mathbb{R} ;\end{cases} \\
2 \mathscr{P}^{\lambda_{2}}(x)-\mathscr{P}^{\lambda}(x) \begin{cases}\leq \gamma_{2}, & x \in i \mathbb{R}, \\
\geq \gamma_{2}, & x \in \operatorname{supp}\left(l-\lambda_{2}\right),\end{cases}
\end{gathered}
$$

where $\mathscr{P}^{\lambda}(x)=\int \ln \frac{1}{t-x} d \lambda$ is the logarithmic potential of the measure $\lambda$, and $d l(z)=|d z|$ is the Lebesgue measure on $i \mathbb{R}$.

\section{GEOMETRY OF THE PROBLEM AND ZEROS DISTRIBUTIONS}

In [3] the main term of asymptotics (6) and the limiting zero distribution (7) for the Pollaczek multiple orthogonal polynomials (5), (4), (2) were obtained by applying the Darboux method to the generating function. Then it was proved in [3] that the limiting measure in (7) indeed satisfies the Nikishin equilibrium relations (10). In this section we consider an inverse problem: we directly find an explicit solution to a boundary value problem that is equivalent to equilibrium relations (10).

Consider the Cauchy transform of the equilibrium measure $\lambda$ :

$$
H(z):=-\hat{\lambda}(z)=\int_{\mathbb{R}} \frac{d \lambda(x)}{x-z} .
$$

"Complexifying" equilibrium relations (10), differentiating the result, and taking the real part, we obtain

$$
\begin{gathered}
\operatorname{Re}(2 \hat{\lambda}(x))-\hat{\lambda}_{2}(x) \\
=\left\{\begin{array}{rll}
-\pi & \text { on } & \mathbb{R}_{-} \cap \operatorname{supp}(\lambda), \\
\pi & \text { on } & \mathbb{R}_{+} \cap \operatorname{supp}(\lambda)
\end{array}\right.
\end{gathered}
$$

and

$$
\operatorname{Re}\left(2 \hat{\lambda}_{2}(x)-\hat{\lambda}(x)\right)=0 \quad \text { on } \operatorname{supp}\left(l-\lambda_{2}\right) .
$$

The first relation implies that the function $H$ has an analytic continuation across the banks of the cuts along $\mathbb{R}_{-} \cap \operatorname{supp}(\lambda)$ and $\mathbb{R}_{+} \cap \operatorname{supp}(\lambda)$. Thus, $H$ can be lifted to the Riemann surface, where

$$
\begin{aligned}
& H(z)=\pi+\hat{\lambda}(z)-\hat{\lambda}_{2}(z):=H_{1}(z), \\
& H(z)=-\pi+\hat{\lambda}(z)-\hat{\lambda}_{2}(z):=H_{2}(z)
\end{aligned}
$$

are continuations to other sheets of the Riemann surface. Let us assume that $H(z)$ is a single-valued function on the three-sheeted Riemann surface: $\mathscr{R}=$ ${\overline{\left\{\mathscr{R}^{(j)}\right.}}_{\}_{j=0}}^{2}, \overline{\mathscr{R}^{(j)}}=\overline{\mathbb{C}}$. Performing the corresponding cuts, we obtain three branches $H=\left\{H_{j}\right\}_{j=0}^{2}$, where $H_{0}(z)=-\hat{\lambda}(z)$ is a holomorphic function in 
$\overline{\mathbb{C}} \backslash \operatorname{supp}(\lambda)$, and (11) and (12) imply that, for $z \rightarrow \infty$, we have

$$
\begin{gathered}
H_{0}(z)=-\frac{2}{z}+\ldots, \quad H_{1}(z)=\pi+\frac{1}{z}+\ldots, \\
H_{2}(z)=-\pi+\frac{1}{z}+\ldots .
\end{gathered}
$$

The function $H$ is sought in the form

$H(\mathbf{z})=\frac{2}{i} \ln \psi(\mathbf{z})$ on $\mathscr{R} \backslash\left\{\mathbf{z} \in \mathscr{R}: \psi(\mathbf{z}) \in \mathbb{R}_{-}\right\}=: \tilde{\mathscr{R}}$,

where $\psi$ is a meromorphic function on some compact three-sheeted Riemann surface $\mathscr{R}$. For $\psi$ (in the case of its existence), relations (14) and (13) give

$$
\psi(\mathbf{z})=\left\{\begin{array}{l}
1-\frac{i}{\mathbf{z}}+\ldots, \quad \mathbf{z} \rightarrow \infty^{(0)}, \\
i-\frac{1}{2 \mathbf{z}}+\ldots, \quad \mathbf{z} \rightarrow \infty^{(1)}, \\
-i+\frac{1}{2 \mathbf{z}}+\ldots, \quad \mathbf{z} \rightarrow \infty^{(2)},
\end{array}\right.
$$

where $q^{(j)}$ denotes a point on $\mathscr{R}^{(j)}$ with the canonical projection onto the plane being $q \in \overline{\mathbb{C}}$. Let us use, as $\psi$, the simplest function mapping $\mathscr{R}$ to $\overline{\mathbb{C}}$. The inverse of this function is a rational function $z=r(\psi)$. From (15) we have

$$
\begin{gathered}
z=-i \frac{\psi(\psi+1)}{\left(\psi^{2}+1\right)(\psi-1)} \\
\Rightarrow \psi^{3}+\frac{i-z}{z} \psi^{2}+\frac{i+z}{z} \psi-1=0 .
\end{gathered}
$$

The discriminant of the algebraic function $\psi(z)$ is equal to $16 z^{4}-44 z^{2}-1$. The function has four branch points $\pm e_{1}$ and $\pm e_{2}$, where

$$
e_{1}=\frac{1}{4} \sqrt{22+10 \sqrt{5}}, \quad e_{2}=\frac{i}{4} \sqrt{-22+10 \sqrt{5}} .
$$

Finally, we conclude that the measure $\lambda(x)$ defined in (11), (14), and (16) is the solution of the equilibrium problem (9); moreover, $\operatorname{supp}(\lambda)=\left[-e_{1}, e_{1}\right]$. For the support of the second measure $\lambda_{2}$ in (9), it holds that supp $\left(l-\lambda_{2}\right)=i \mathbb{R} \backslash\left(-e_{2}, e_{2}\right)$. At the same time, $\lambda$ is the limit of the zeros counting measure (7).

Now we present the main term of asymptotics (6) in explicit form. Consider an abelian integral defined on the open Riemann surface $\tilde{\mathscr{R}}$ :

$$
G=\int H d z \text {. }
$$

It can be computed using uniformization (16) for $\mathscr{R}$ :

$$
G(z)=\text { const }+\mathbf{z} H(\mathbf{z})+2 \ln \left(\frac{\psi(\mathbf{z})-1}{\psi^{2}(\mathbf{z})+1}\right) .
$$

If we fix an additive constant such that $\operatorname{Re} G_{0}+\operatorname{Re} G_{1}=$ $\operatorname{Re} G_{2}=0$, then we define the unique function (on $\tilde{\mathscr{R}}$ )

$$
\Phi(\mathbf{z})=\exp \{-G(\mathbf{z})\},
$$

and $\left|\Phi_{0}\right|$ is the main term of the asymptotics in (6).

We emphasize that the expressions obtained here for the right hand sides of (6) and (7) agree with the results of [3].

\section{STRONG ASYMPTOTICS}

In this section we state the main result of this paper-a theorem on the strong asymptotics.

To refine the main asymptotic term in the formulas for the strong asymptotics, we define a multiplicative function, namely, the Szegé function. For this purpose, we set

$$
\zeta=\frac{\psi+\psi^{-1}}{2}
$$

Then, from (16), it follows that

$$
z^{2}=\frac{1+\zeta}{4 \zeta^{2}(1-\zeta)}
$$

and, for the algebraic function $\zeta(z)$, we fix the holomorphic branch (in $\overline{\mathbb{C}} \backslash\left[-e_{1}, e_{1}\right]$ )

$$
\zeta_{1}(z)=1+O\left(\frac{1}{z^{2}}\right)
$$

The role of the Szegé function is played by

$$
\mathscr{H}\left(\zeta_{1}(z)\right), \text { where } \mathscr{H}(\zeta)=\frac{\zeta}{2}\left(\frac{1+\zeta}{\zeta^{2}+\zeta-1}\right)^{1 / 2}
$$

is the holomorphic branch in $\left(-\infty, \frac{-1+\sqrt{\zeta}}{2}\right)$ with normalization $\mathscr{H}(1)=1$. The following result holds.

Theorem 1. For the sequences of Pollaczek scaled multiple orthogonal polynomials (5), (4), (2), the following strong asymptotics formulas as $n \rightarrow \infty$ are valid.

$$
\begin{aligned}
& \text { For } z \in \mathbb{C} \backslash\left[-e_{1},+e_{1}\right] \text {, } \\
& Q_{n}(z)=\Phi_{0}^{n}(z) \mathcal{H}\left(\zeta_{1}(z)\right)\left(1+\mathcal{O}\left(\frac{1}{n\left(\left|z^{2}\right|+1\right)}\right)\right)
\end{aligned}
$$

locally uniformly outside $\left[-e_{1},+e_{1}\right]$.

For compact subsets of $\left(-e_{1},+e_{1}\right)$,

$$
\begin{aligned}
& Q_{n}(x)=\left(\Phi_{0+}^{n}(z) \mathcal{H}\left(\zeta_{1+}(x)\right)\right. \\
& \left.+\Phi_{0-}^{n}(z) \mathcal{H}\left(\zeta_{1-}(x)\right)+\mathcal{O}\left(\frac{1}{n}\right)\right) .
\end{aligned}
$$




\section{MATRIX RIEMANN-HILBERT PROBLEM}

Here, we highlight the main steps in the derivation of the strong asymptotics formulas (19) and (21).

It is well known that multiple orthogonal polynomials are characterized by the following matrix RiemannHilbert problem. For $n \in \mathbb{N}$, find a $3 \times 3$ matrix-valued function $\mathbf{Y}$ that is analytic in $\mathbb{C} \backslash \mathbb{R}$ and has continuous boundary values such that:

(RH-Y1) for $x \in(\infty,+\infty)$ the boundary condition

$$
\mathbf{Y}_{+}(x)=\mathbf{Y}_{-}(x)\left(\begin{array}{ccc}
1 & w_{1, n}(x) & w_{2, n}(x) \\
0 & 1 & 0 \\
0 & 0 & 1
\end{array}\right)
$$

is satisfied;

$(\mathrm{RH}-\mathrm{Y} 2) \mathbf{Y}(z)=\left(\mathbf{I}+\mathbf{O}\left(z^{-1}\right)\right) \operatorname{diag}\left(z^{2 n}, z^{-n}, z^{-n}\right)$, as $z \rightarrow \infty, z \in \mathbb{C} \backslash \mathbb{R}_{-}$.

This problem has a unique solution, which can be expressed in terms of the polynomials $Q_{n}(z)$. Specifically, the first row of the matrix $\mathbf{Y}$ is

$$
\begin{gathered}
\left(Q_{n}(z), \frac{1}{2 \pi i} \int_{0}^{+\infty} \frac{Q_{n}(x) w_{1, n}(x) d x}{x-z},\right. \\
\left.\frac{1}{2 \pi i} \int_{0}^{+\infty} \frac{Q_{n}(x) w_{2, n}(x) d x}{x-z}\right) .
\end{gathered}
$$

The desired asymptotic formulas are sought in the form of an approximate solution to this boundary value problem for large $n$. For this purpose, by applying a chain of equivalent transformations, the matrix $\mathbf{Y}$ is transformed into a matrix-valued function that is analytic in $\overline{\mathbb{C}}$, except for a system of contours where the jump in its boundary values tends to an identity matrix as $n \rightarrow \infty$. Then the resulting matrix itself tends to an identity matrix uniformly in $\overline{\mathbb{C}}$. Inverting this chain of equivalent transformations, we obtain an approximate representation of $\mathbf{Y}$ for large $n$ and, therefore, we obtain the desired asymptotic formulas. Let us present the key transformations of this chain in our case.

The preliminary transformation is $\mathbf{Y} \rightarrow \mathbf{X}$. Define

$$
D^{(L)}:=\left(\begin{array}{ccc}
1 & 0 & 0 \\
0 & \frac{1}{\sqrt{z}} & -\frac{1}{\sqrt{z}} \sinh (\pi n z) e_{n} \\
0 & -\sqrt{z} & \sqrt{z} \cosh (\pi n z) e_{n}
\end{array}\right) ;
$$

$$
D^{(R)}:=\left(\begin{array}{ccc}
1 & 0 & 0 \\
0 & \frac{1}{\sqrt{z}} & -\frac{1}{\sqrt{z}} \sinh (\pi n z) e_{n}^{-1} \\
0 & \sqrt{z} & \sqrt{z} \cosh (\pi n z) e_{n}^{-1}
\end{array}\right),
$$

where $e_{n}:=e_{n}(z):=\exp \{\pi n z\}$. Define the matrix function

$$
\begin{aligned}
& \mathbf{X}:= \begin{cases}\mathbf{Y} D^{(L)} & \text { on } i \mathbb{R} \times \mathbb{R}^{(-)}, \\
\mathbf{Y} D^{(R)} & \text { on } i \mathbb{R} \times \mathbb{R}^{(+)},\end{cases} \\
& \text {then } \quad \mathbf{X}_{+}=\mathbf{X}_{-} J_{1} \text { on } i \mathbb{R} \cup \mathbb{R} .
\end{aligned}
$$

Then the jump along the real axis becomes

$$
\begin{aligned}
& J_{1}=\left(\begin{array}{ccc}
1 & \frac{4(z)^{1 / 2}}{e_{n}^{3}-e_{n}^{-1}} & 0 \\
0 & 1 & 0 \\
0 & 0 & 1
\end{array}\right) \text { on } \mathbb{R}^{(-)}, \\
& J_{1}=\left(\begin{array}{ccc}
1 & \frac{-4(z)^{1 / 2}}{e_{n}^{-3}-e_{n}^{+1}} & 0 \\
0 & -1 & 0 \\
0 & 0 & -1
\end{array}\right) \text { on } \mathbb{R}^{(+)} .
\end{aligned}
$$

Additionally, a new jump appears along the imaginary axis:

$$
J_{1}=\left(\begin{array}{ccc}
1 & 0 & 0 \\
0 & e_{n}^{-2} & 0 \\
0 & -2 & e_{n}^{2}
\end{array}\right) \text { on } i \mathbb{R},
$$

which, on the set $\left[-i \infty,-e_{2}\right] \cup\left[e_{2}, i \infty\right]$, has a local factorization as a product of three matrices. The two extremes tend to the identity matrix as $n \rightarrow \infty$ (in the corresponding half-planes) and the central matrix does not depend on $n$.

The next transform is $\mathbf{X} \rightarrow \mathbf{Z}$ :

$$
\begin{gathered}
\mathbf{Z}:=C \mathbf{X} S, \quad S:=\operatorname{diag}\left(\Phi_{0}^{n}, \Phi_{1}^{n} \tilde{e}_{n}^{-1}, \Phi_{2}^{n} \tilde{e}_{n}\right), \\
\tilde{e}_{n}:=\left\{\begin{array}{l}
e_{n} \text { on } \mathbb{R}^{(-)} \times i \mathbb{R}, \\
e_{n}^{-1} \text { on } \mathbb{R}^{(+)} \times i \mathbb{R} .
\end{array}\right.
\end{gathered}
$$

After locally factorizing the jump of the matrix $\mathbf{Z}$ across $\left[-e_{1},+e_{1}\right]$ (as a product of three matrices as described above), we obtain a boundary value problem for the matrix valued function

$$
\begin{gathered}
\mathbf{N} \in H\left(\mathbb{C} \backslash\left\{i \mathbb{R} \backslash\left[e_{2},-e_{2}\right]\right\} \backslash\left[-e_{1},-\infty\right]\right), \\
C\left(\left\{i \mathbb{R} \backslash\left[e_{2},-e_{2}\right] \cup\left[-e_{1}, \infty\right]\right\}\right),
\end{gathered}
$$

with the jump $N_{+}=N \_J_{3}$ on $\left\{i \mathbb{R} \backslash\left[e_{2},-e_{2}\right] \cup\left[-e_{1}, \infty\right]\right\}$, which does not depend on $n$ : 


$$
\begin{aligned}
& J_{3}=\left(\begin{array}{ccc}
0 & -4(z)^{1 / 2} & 0 \\
4(z)^{-1 / 2} & 0 & 0 \\
0 & 0 & -1
\end{array}\right) \text { on }\left[-e_{1}, 0\right] \text {, } \\
& J_{3}=\left(\begin{array}{ccc}
1 & 0 & 0 \\
0 & 0 & \frac{1}{2} \\
0 & -2 & 0
\end{array}\right) \text { on } i \mathbb{R} \backslash\left[e_{2},-e_{2}\right], \\
& J_{3}=\left(\begin{array}{ccc}
0 & -4(z)^{1 / 2} & 0 \\
-4(z)^{-1 / 2} & 0 & 0 \\
0 & 0 & -1
\end{array}\right) \text { on }\left[0, e_{1}\right] \text {, } \\
& J_{3}=\left(\begin{array}{ccc}
1 & 0 & 0 \\
0 & -1 & 0 \\
0 & 0 & 1
\end{array}\right) \text { on }\left[e_{1}, \infty\right] \text {. }
\end{aligned}
$$

The solution of this boundary value problem defines the Szegó function.

The final transformation uses $\mathbf{N}$ and the solution of local boundary value problems around the branch points and the origin in order to get a matrix with a jump close to the identity matrix.

Detailed proofs of the results presented above can be found in [4].

\section{ACKNOWLEDGMENTS}

The first author acknowledges the support of the Russian Science Foundation, project no. 14-21-00025.
The second author's research was supported by MTM2012-36732-C03-01 MEC. The third author's research was supported by MTM2011-28952-C02-01 ERDF, Exc. Grant no. P11-FQM-7276, Res. Group FQM-229, and CEIMAR Universidad de Almeria.

\section{REFERENCES}

1. F. Pollaczek, C. R. Acad. Sci. Paris 228, 1363-1365 (1949).

2. B. Beckermann, J. Coussement, and W. Van Assche, J. Approx. Theory 132, 155-181 (2005).

3. V. N. Sorokin, Sb. Math. 200 (4), 577-595 (2009).

4. A. I. Aptekarev, G. López Lagomasino, and A. Martínez-Finkelshtein, arXiv:1410:1261.

5. A. I. Aptekarev, P. M. Bleher, and A. B. J. Kuijlaars, Commun. Math. Phys. 259 (2), 367-389 (2005).

6. A. B. J. Kuijlaars, A. Martínez-Finkelshtein, and F. Wielonsky, Commun. Math. Phys. 286 (1), 217-275 (2009).

7. A. I. Aptekarev, V. G. Lysov, and D. N. Tulyakov, Sb. Math. 202 (2), 155-206 (2011).

8. A. B. J. Kuijlaars, Proceedings of the International Congress of Mathematicians (Hindustan Book Agency, New Delhi, 2010), Vol. 3, pp. 1417-1432.

9. A. I. Aptekarev and A. B. J. Kuijlaars, Russ. Math. Surv. 66 (6), 1133-1199 (2011).

10. E. M. Nikishin, Math. USSR-Sb. 41 (4), 409-425 (1982).

11. A. A. Gonchar, E. A. Rakhmanov, and V. N. Sorokin, Sb. Math. 188 (5), 671-696 (1997).

12. A. I. Aptekarev and V. G. Lysov, Sb. Math. 201 (2), 183-234 (2010).

13. A. B. J. Kuijlaars, M. Bender, and S. Delvaux, J. Approx. Theory 163, 1606-1637 (2011).

14. A. I. Aptekarev, G. López Lagomasino, and A. Martínez-Finkelshtein, arXiv:1403:3729. 